\title{
Aplicación del pesaje de vehículos en movimiento al dimensionamiento de firmes. Influencia de la precisión de los pesajes
}

\section{Application of WIM to pavement design. Effect of WIM accuracy}

\author{
J. Leal Bermejo $^{(*)}$, J. M. Pardillo Mayora ${ }^{(* *)}$
}

\section{RESUMEN}

\begin{abstract}
El pesaje de vehículos en movimiento permite conocer los pesos de los vehículos y sus ejes cuando circulan a su velocidad normal de recorrido. En este artículo se describe su aplicación al dimensionamiento de firmes viarios y se estudia la influencia de la precisión de los datos obtenidos mediante el pesaje dinámico en el cálculo de la vida útil de los firmes. Para ello se describen, primeramente, los principales factores que afectan a la precisión del pesaje en movimiento, en especial, la interacción entre el vehículo y el pavimento. Luego, se presenta un ensayo realizado para comprobar el efecto de la precisión de los pesajes dinámicos en la estimación de la vida útil de los firmes. Los resultados obtenidos indican que los errores de las mediciones de pesos se incrementan notablemente al determinar la vida útil del firme.
\end{abstract}

Palabras clave: pesaje en movimiento; pesaje dinámico; pesos de los vehículos; cargas de ejes; dimensionamiento de firmes.

\begin{abstract}
Weigh-in-motion (WIM) techniques can provide data on vehicle and axle weights when they are travelling at full highway speeds. In this paper, the application of WIM data to the design of road pavements is described and the effect of WIM load data accuracy on the estimate of pavement service life is analysed. Thus, the main factors affecting WIM accuracy are explained, especially those related to vehicle-pavement interaction. Afterwards, a trial that has been conducted to evaluate the effect of WIM load data accuracy on pavement life prediction will be presented. The results obtained show that the errors in axle load measurements can lead to higher errors in pavement life estimates.
\end{abstract}

Keywords: weigh-in motion; WIM; vehicle weights; axle loads; pavement design.

(*) CEDEX, Centro de Estudios del Transporte. Madrid (España).

(*) Universidad Politécnica de Madrid, ETSICCP. Madrid (España).

Persona de contacto/Corresponding author: jleal@cedex.es (J. Leal Bermejo)

ORCID: http://orcid.org/oooo-0oo2-4435-2820 (J. Leal Bermejo); http://orcid.org/oooo-ooo2-7327-9511

(J. M. Pardillo Mayora)

Cómo citar este artículo/Citation: Leal Bermejo, J., Pardillo Mayora, J. M. (2016). Aplicación del pesaje de vehículos en movimiento al dimensionamiento de firmes. Influencia de la precisión de los pesajes. Informes de la Construcción, 69(545): e181, doi: http://dx.doi. org/10.3989/ic.16.054.

Copyright: (c) 2017 CSIC. Licencia / License: Salvo indicación contraria, todos los contenidos de la edición electrónica de Informes de la Construcción se distribuyen bajo una licencia de uso y distribución Creative Commons Attribution License (CC BY) Spain 3.o. 


\section{INTRODUCCIÓN}

El pesaje de vehículos en movimiento o pesaje dinámico, normalmente conocido por sus siglas en inglés WIM (Weigh-In-Motion) abarca una serie de tecnologías y procedimientos dirigidos a evaluar los pesos de los vehículos en la carretera mediante la medición de las fuerzas de impacto o cargas dinámicas en los puntos de apoyo (ruedas) de cada eje de los vehículos en movimiento, operación que debe ser realizada en condiciones normales de circulación y sin perturbar el tráfico rodado.

La aplicación del pesaje en movimiento a la optimización del dimensionamiento de firmes presenta claras ventajas, debido a que, por un lado, estos sistemas pueden proporcionar datos muy detallados sobre las cargas del tráfico que deben resistir, $\mathrm{y}$, por otra parte, a que pueden ser utilizados para facilitar información relativa al tráfico pesado real de cada carretera en particular, lo que permite un dimensionamiento más ajustado en comparación con el que podría realizarse utilizando unos valores dados por defecto en las normas.

Para que el pesaje en movimiento pueda cumplir adecuadamente esta función, se deben estudiar los factores que influyen en su funcionamiento y precisión, con vistas a evaluar el efecto que tienen estos factores en la determinación de la vida útil del firme. Para ello, además, se ha realizado un ensayo de pesaje a una pequeña muestra de vehículos en la carretera, en el que se ha determinado la precisión de los pesos obtenidos y la influencia de ésta en la determinación de la vida útil de los firmes. Por último, como comparación, se pasa revista a una serie de investigaciones sobre el efecto que tendría la precisión de los pesajes si se utilizaran métodos mecánicoempíricos de dimensionamiento.

\section{LA TECNOLOGÍA DEL PESAJE EN MOVIMIENTO}

El pesaje dinámico o de vehículos en movimiento surgió en la década de los años 60 y 70 , pero no fue hasta finales de la década de los 80 cuando las administraciones de carreteras comenzaron a utilizarlo de una forma más o menos generalizada. Tras diferentes proyectos de investigación en décadas pasadas, esta técnica ha alcanzado actualmente su madurez, que se ha traducido en la existencia de normativas en diferentes áreas geográficas del mundo que regulan los procedimientos de pesaje, las condiciones que deben cumplir los emplazamientos de los equipos y los métodos de calibración y de evaluación de la precisión de estos. Entre estos documentos normativos se pueden destacar las Especificaciones Europeas del Pesaje en Movimiento elaboradas en el marco de la Acción COST 323 (1) y la norma estadounidense ASTM E-1318 (2). En las Especificaciones Europeas del COST 323 se ofrece una clasificación de la precisión de los sistemas de pesaje muy utilizada actualmente, en la que cada clase viene definida por una letra y un número entre paréntesis [es decir $\mathrm{A}(5), \mathrm{B}(10), \mathrm{C}(15), \mathrm{D}(20), \mathrm{E}(25)]$, que representa el intervalo de confianza de los equipos de esa clase para medir el peso de aproximadamente el $95 \%$ de los vehículos.

\subsection{Estructura de un sistema de pesaje en movimiento}

En general, un sistema de pesaje de vehículos en movimiento consta de los siguientes elementos (véase la Figura 1):
- Sensores de peso, instalados en uno o varios carriles.

- Detectores de presencia (normalmente, lazos de inducción), utilizados para detectar la presencia de un vehículo y activar el sistema, así como para medir la velocidad y la longitud de los vehículos.

- Aparato electrónico de control del funcionamiento del sistema, que además efectúa el procesamiento de las señales enviadas por los sensores y detectores.

\subsection{Tecnologías de pesaje en movimiento}

Existen diferentes tecnologías de pesaje en movimiento, entre las que se pueden citar las siguientes como las más comunes:

- Los sensores piezoeléctricos, entre los cuales existen las tecnologías de los sensores piezocerámicos, los piezopolímeros y los de cristal de cuarzo.

- Las placas en flexión.

- Las esteras capacitivas, muy utilizadas en instalaciones temporales de pesaje.

- Los puentes de pesaje en movimiento (en inglés, BridgeWIM) que consisten en puentes de carretera cuyo tablero ha sido instrumentado en su parte inferior con bandas extensométricas, para medir su deformación al paso de los vehículos.

\subsection{Precisión de las diferentes tecnologías de pesaje en movimiento}

A lo largo de los años se han realizado numerosos ensayos para verificar la precisión de cada una de las tecnologías mencionadas. Los resultados obtenidos para cada tecnología de pesaje dinámico en algunos de estos ensayos han sido los siguientes:

- Sistemas con sensores piezoeléctricos: su precisión varía en función de las distintas tecnologías y métodos de autocalibración, pudiendo alcanzar los piezocerámicos hasta un \pm $10 \%$ para el 95\% de los vehículos (3), (4), lo que representa una clase B(10) de las Especificaciones Europeas WIM del COST 323, y los cristales de cuarzo valores aún mejores [clase $\mathrm{B}(7)$ ], que además son insensibles a las variaciones de la temperatura, al contrario que los sensores piezocerámicos y piezopolímeros (5).

- Placas en flexión: alcanzan una precisión $\pm 10 \%$ para el $95 \%$ de los vehículos.

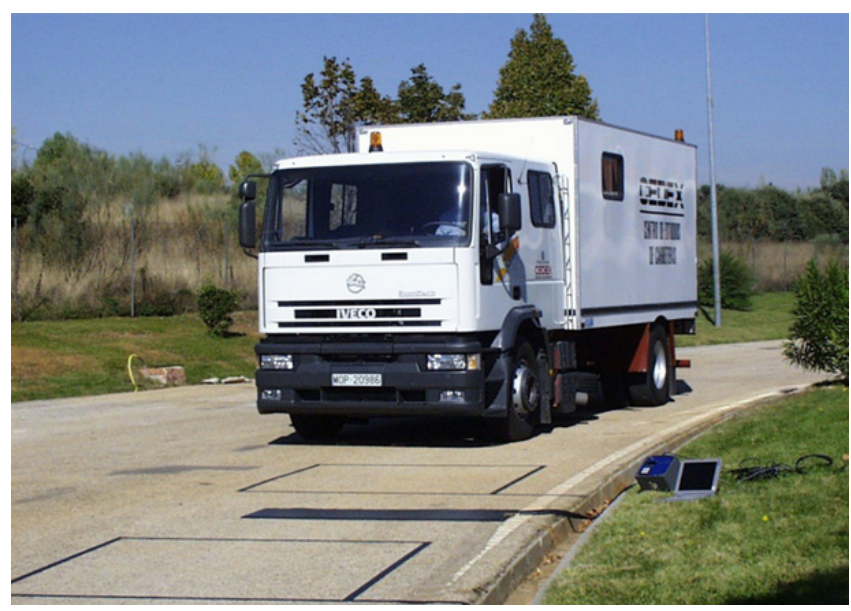

Figura 1. Sistema de pesaje dinámico portátil utilizado por el CEDEX y camión tarado para la calibración. 
- Esteras capacitivas: en instalación portátil pueden medir el peso del $95 \%$ de los vehículos dentro de un intervalo del $\pm 25 \%(6)$, mientras que en instalación fija alcanzan aproximadamente un $\pm 10 \%$ para el $95 \%$ de los vehículos (3).

- Sistemas de puentes de pesaje en movimiento (sistemas Bridge-WIM): pueden alcanzar precisiones de aproximadamente hasta un $\pm 10 \%$ para el $95 \%$ de los vehículos, lo que es aproximadamente la clase B(10) de las Especificaciones Europeas WIM del COST 323 en puentes losa de hormigón (7), pero su precisión es algo menor [Clase C(15) de las Especificaciones Europeas WIM] cuando se instrumentan puentes metálicos (8).

\section{FACTOR PRINCIPAL QUE AFECTA A LA PRECISIÓN DEL PESAJE EN MOVIMIENTO: LA INTERACCIÓN DINÁMICA ENTRE VEHÍCULO Y PAVIMENTO}

Los vehículos en su movimiento ejercen a través de los neumáticos una fuerza sobre el pavimento que se desplaza a su misma velocidad y que tiene tres componentes (9):

- Una componente estacionaria (constante) de tipo estático.

- Una componente móvil que se puede considerar constante en el tiempo.

- Una componente móvil variable en el tiempo, de tipo dinámico, debida a la vibración del vehículo al ser excitado por las irregularidades del firme.

La componente estacionaria es la distribución del peso estático del vehículo entre los distintos ejes y ruedas, tal como se haría en una superficie horizontal. La componente móvil constante es debida a los diferentes mecanismos de transferencia de las cargas estáticas de unos ejes y ruedas a otros. Estos mecanismos de transferencia ocurren muy lentamente en relación con las frecuencias dinámicas del vehículo y tienen lugar en dos direcciones básicas:

- Longitudinalmente, entre distintos ejes, que tienen lugar cuando el vehículo se mueve en un tramo en pendiente, con transferencia de la carga hacia el eje situado a una altura inferior.

- Transversalmente al vehículo, entre ruedas de un mismo eje, debido a la diferencia de altura entre éstas, por causa del peralte, pendiente transversal de la calzada o simplemente por baches.

Puede decirse que estas componentes tienen poca influencia sobre los datos obtenidos en los pesajes dinámicos, pues precisamente se suele exigir la instalación del sistema de pesaje en un terreno llano y recto, con objeto de evitar en lo posible estos efectos.

Por último, las características del movimiento vibratorio debidas a la componente de tipo dinámico son las siguientes (10):

1. La masa suspendida del vehículo (chasis, cabina, caja y carga) vibra con la frecuencia de la suspensión, entre 1,5 y $4 \mathrm{~Hz}$ (a velocidades normales de circulación, la longitud de onda oscila entre 7 y $19 \mathrm{~m}$ ), dependiendo el valor concreto del tipo de suspensión, del amortiguamiento de ésta y del peso por eje (cuanto más cargado va el vehículo, menor frecuencia de oscilación).
2. La masa no suspendida (ejes y ruedas) oscila con una frecuencia entre 10 y $15 \mathrm{~Hz}$ (la longitud de oscilación puede variar entre 2 y 4 m), debida la rigidez de los neumáticos.

Generalmente, para pavimentos que no están en muy mal estado, la contribución de la masa suspendida a las fuerzas dinámicas es mucho mayor que la de la no suspendida, por ello se suele considerar su modo de vibración como el modo fundamental de oscilación de las cargas dinámicas del vehículo.

Esta oscilación se suele aproximar por una función de tipo senoidal con una componente aleatoria superpuesta. La amplitud de la onda es variable (véase la Figura 2), y con una frecuencia de oscilación constante en el tiempo, que depende de la suspensión del vehículo.

Las cargas dinámicas máximas que el vehículo ejerce sobre el pavimento vendrán dadas por la amplitud de esta onda, cuya magnitud depende de (11):

- la irregularidad superficial del pavimento;

- la velocidad del vehículo, y

- las características de la suspensión del vehículo.

En algunos estudios se ha tratado de evaluar el valor de la amplitud de estas oscilaciones, como el llevado a cabo por S. Ma y M. Caprez (12), que determinó que la amplitud de oscilación puede llegar a un $40 \%$ o un $50 \%$ de la carga estática en pavimentos en estado deficiente con PSI $=2$, debiendo de tener el pavimento como mínimo un PSI $=3,5$ para que estas cargas dinámicas no superen el $15 \%$ para una velocidad del vehículo de $80 \mathrm{~km} / \mathrm{h}$.

El problema que plantea esta circunstancia proviene de que el sensor de peso queda instalado en un punto determinado de la carretera, y solamente podrá medir la carga aplicada por el vehículo en ese lugar, que constituye un punto de la función de variación en el espacio de su carga dinámica, pudiendo apartarse bastante de su peso estático (alrededor de un $\pm 20 \%$ de su valor en firmes en buen estado), que es normalmente la magnitud que se pretende medir.
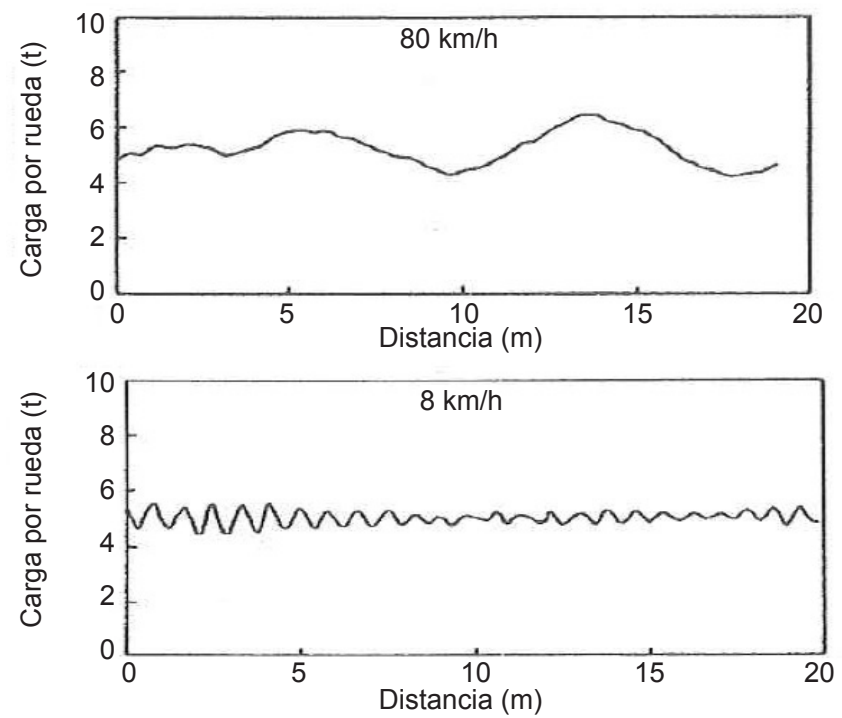

Figura 2. Variación de las cargas de un vehículo a diferentes velocidades. Fuente: (11) 
Como consecuencia, aunque los anteriores efectos se pueden corregir en gran medida con una adecuada calibración, será de gran importancia instalar los sensores en un pavimento cuyas características superficiales sean suficientemente buenas como para acotar los efectos dinámicos de las cargas y de esta forma mejorar la exactitud de sus medidas.

\section{APLICACIÓN DEL PESAJE EN MOVIMIENTO AL PROYECTO DE FIRMES}

\subsection{Consideración de las cargas del tráfico en el dimensionamiento de firmes}

Las solicitaciones del tráfico son uno de los factores de entrada (junto con las características de los materiales y las condiciones climáticas) en el dimensionamiento de un firme.

Para analizar el efecto de las cargas del tráfico sobre el firme es necesario conocer el número de veces que se van a repetir estas cargas sobre la vida de servicio del firme, lo que viene dado por el número de ejes (y, por tanto, con el número de vehículos) que van a circular por la sección de proyecto durante su vida útil, así como la distribución de frecuencias de los distintos pesos por eje.

El procedimiento que se sigue en el dimensionamiento para considerar el efecto de las sucesivas cargas del tráfico de distinta magnitud se basa en la aplicación de dos supuestos (13):

1. La Ley de Miner en cuanto a la acumulación de los efectos de las cargas, que establece que la fatiga producida por la aplicación de una carga es independiente de todas las solicitaciones anteriores, o sea, que el daño acumulado por un conjunto aleatorio de cargas es la suma de los deterioros parciales de cada una de ellas.

Por tanto, si un firme es capaz de soportar $N_{i}$ aplicaciones de una carga de valor $q_{i}$ antes de fisurarse, de acuerdo con la hipótesis de Miner, la fatiga $f_{i}$ (o deterioro elemental, $D_{i}$ ) provocada por una aplicación aislada de la carga $q_{i}$, será:

$$
f_{i}=D_{i} \frac{1}{N_{i}}
$$

El grado de fatiga total causado por un conjunto aleatorio de cargas es igual a la acumulación de los deterioros parciales de cada una de ellas, es decir:

$$
F=\sum_{i} f_{i} \cdot n_{i}=\sum_{i} D_{i} \cdot n_{i}=1
$$

Siendo:

$F=$ Grado total de fatiga por un conjunto de cargas (se cumplirá $0 \leq F \leq 1$ ).

$n_{i}=$ número de aplicaciones de la carga $q_{i}$.

$f_{i}=D_{i}$ fatiga elemental o deterioro elemental provocado por una aplicación aislada de la carga $q_{i}$.

2. En cuanto a los efectos destructivos de las cargas de distinta magnitud, se toma la equivalencia de deterioros ocasionados en el firme causados por las distintas cargas obtenida en el ensayo AASHO, realizado a principios de los años 6o, en el que se comprobó experimentalmente que la relación de equivalencia de deterioros entre cargas de distinta magnitud sigue una ley exponencial:

$$
e=D_{i} / d=\left(P_{i} / P_{o}\right)^{a}
$$

siendo $e$ la equivalencia unitaria a efectos de deterioro producido en el firme entre la carga $P_{i}$ y la $P_{o}$ adoptada como eje tipo (en España es $128 \mathrm{kN}$ ), $D_{i}$ el deterioro producido por el eje de peso $P_{i}$, y $d$ el deterioro producido por el eje tipo de peso $P_{o}$.

Desde entonces el procedimiento normal de calcular la agresividad en relación con el firme de todo un conjunto o espectro de cargas del tráfico de distinta magnitud ha sido considerar la equivalencia (de deterioros) de cada una de ellas en relación con la carga tipo de referencia mediante la aplicación de la fórmula [2] obtenida en el ensayo AASHO ( $u$ otra de tipo similar).

Si se sustituye en esta expresión el deterioro $D_{i}$ por

$$
D_{i}=d \cdot\left(\frac{p_{i}}{128}\right)^{\alpha} \quad \text { para los ejes simples. }
$$

Esta relación es esencialmente válida para los ejes simples, mientras que para los ejes tándem y trídem es preciso tener en cuenta la superposición de efectos de los ejes individuales que los forman, utilizándose para ello un coeficiente $K$, que representa la forma en la que se superponen estos efectos.

$$
\begin{aligned}
D_{j} & =d \cdot\left(\frac{p_{j}}{128}\right)^{\alpha} \cdot K_{T} \quad \text { para los ejes tándem. } \\
D_{k} & =d \cdot\left(\frac{p_{k}}{128}\right)^{\alpha} \cdot K_{T r} \quad \text { para los ejes trídem. }
\end{aligned}
$$

Siendo $d$ el deterioro ocasionado por el eje tipo (128 kN).

En el momento de rotura por fatiga del firme se cumplirá [1]:

$$
\sum n_{i} \cdot D_{i}=\sum n_{e q} \cdot d=1
$$

Siendo $n_{e q}$ el número de ejes tipo equivalentes que producen el mismo deterioro o grado de fatiga.

Desglosando la anterior expresión según el tipo de eje, quedaría:

$$
\sum_{i} n_{i} \cdot\left(\frac{P_{i}}{128}\right)^{\alpha} \cdot d+\sum_{j} n_{j} \cdot\left(\frac{P_{j}}{128}\right)^{\alpha} \cdot d \cdot K_{T}+\sum_{k} n_{k} \cdot\left(\frac{P_{k}}{128}\right)^{\alpha} \cdot d \cdot K_{T r}=1
$$

Entonces se puede deducir el número $n_{e q}$ de ejes tipo $(128 \mathrm{kN})$ equivalentes al conjunto de ejes simples, tándem y trídem considerados:

$$
n_{e q}=\frac{1}{d}=\sum_{i} n_{i} \cdot\left(\frac{P_{i}}{128}\right)^{\alpha}+K_{T} \cdot \sum_{j} n_{j} \cdot\left(\frac{P_{j}}{128}\right)^{\alpha}+K_{T r} \cdot \sum_{k} n_{k} \cdot\left(\frac{P_{k}}{128}\right)^{\alpha}
$$

Con lo cual, el número de ejes tipo equivalentes, $n_{e q}$ puede calcularse fácilmente a partir de los resultados de los pesajes.

El tráfico, que se compone de diferentes vehículos y cargas, se reduce de esta forma a un número de pasadas acumuladas de un eje con la carga de referencia. Se supone que este tráfico equivalente produciría el mismo deterioro (por fatiga o por deformaciones permanentes) que la combinación real de las diferentes cargas del tráfico. Por otro lado, no se considera 
a la vida de servicio como un concepto de tiempo, sino expresada en términos de cargas aplicadas a la estructura del firme (14).

El adelanto más significativo en la pasada década fue la aparición en Estados Unidos de la Mechanistic-Empirical Pavement Design Guide (MEPDG) de la AASTHO (15), que ha abandonado el enfoque de reducir todas las cargas a la carga tipo o patrón, mediante la ley exponencial obtenida empíricamente en el ensayo AASHO, para pasar a utilizar un planteamiento basado en principios de tipo mecánico-empírico, en el que se considera el espectro de cargas completo, calculando las respuestas del pavimento (tensiones, deformaciones y deflexiones) a cada carga y los deterioros parciales producidos. Luego, el procedimiento relaciona empíricamente el deterioro acumulado en el tiempo con los deterioros observados en el firme. Además, se tiene en cuenta el tipo de deterioro y criterio de fallo.

Con este planteamiento se utilizan básicamente los mismos datos del tráfico que para el cálculo de los ejes equivalentes, la diferencia está solamente en que no se convierten las cargas en cargas tipo, sino que mantiene los datos por configuración de ejes y peso.

\subsection{Métodos de considerar el tráfico en el proyecto de firmes}

El procedimiento seguido hasta hace pocos años en las normas de dimensionamiento de la mayoría de los países consistía en que, a partir de datos de pesajes estáticos (por tanto basándose en datos sobre muestras muy pequeñas), se conocía una equivalencia media entre vehículo pesado y eje tipo, que era extrapolada a las carreteras de toda la red, lo que podía conducir a errores importantes en muchos lugares. Por otro lado, para proyectar el firme de un cierto tramo de carretera se instalaba un equipo de aforo automático corriente, capaz de distinguir entre vehículos ligeros y pesados, con el fin de conocer el número de camiones que pasaban durante un cierto tiempo. Entonces, multiplicando el número de vehículos pesados obtenido en esa carretera por el factor de equivalencia medio de cada vehículo pesado en ejes tipo, se obtenía el «tráfico total equivalente».

Una cierta mejora de esta poco precisa estimación se llevó a cabo en ciertos países, como el Reino Unido y Francia, asignando a cada tipo diferente de camión una equivalencia específica en ejes tipo (en vez de asignar el mismo factor de equivalencia para todos los vehículos pesados), obtenida a partir de pesajes estáticos. Así, según los datos obtenidos en esos pesajes en el Reino Unido, por ejemplo, para los vehículos articulados de 5 ejes que circulaban por autopistas resultó una equivalencia de unos 2,10 ejes tipo de $80 \mathrm{kN}$ (16).

Este método es más aproximado, pero tiene el inconveniente de que es necesario conocer la composición detallada del tráfico pesado en ese lugar, dividida en 8 o 9 clases de vehículos, lo cual no se puede lograr con los usuales aparatos automáticos de aforo y requería la realización de contajes manuales.

La siguiente mejora para estimar de forma más fiable las cargas del tráfico total que debe soportar un firme es efectuar su medición directa en el lugar mediante la instalación de un sistema de pesaje dinámico, durante el tiempo que se considere necesario, lo que permite obtener el espectro completo de cargas que soporta.
Por ello, las normas de dimensionamiento de firmes de los distintos países van incorporando la exigencia de realizar pesajes dinámicos a la hora de efectuar el proyecto de un firme nuevo o el refuerzo de uno existente, como ocurre en la norma MEPDG (15) de la AASTHO.

En el Anejo (17) dedicado a las cargas del tráfico de la MEPDG, donde no sea viable realizar un pesaje, se admite instalar un equipo contador-clasificador de vehículos que permita distinguir las proporciones de las distintas clases de vehículos en el tráfico de dicha estación. Al mismo tiempo, para complementar esta información, proporciona una serie de distribuciones de las frecuencias de los distintos tipos de ejes por intervalos de pesos, para cada clase de vehículo y para el conjunto del tráfico total de vehículos pesados, en el caso de disponer sólo de datos de aforadores con clasificación en vehículos ligeros y pesados.

\section{ENSAYO DE COMPROBACIÓN DE LA INFLUENCIA DE LA PRECISIÓN DEL PESAJE EN EL NÚMERO DE EJES EQUIVALENTES}

Para comprobar el efecto de la exactitud y precisión del pesaje dinámico en la estimación del número de ejes equivalentes que debe soportar el firme de una carretera (y, por tanto, de su vida útil), se realizó en la semana del 15 al 19 de junio de 2015 un ensayo en la autopista A-2, pk 82, en Trijueque (Guadalajara).

En el ensayo la Guardia Civil desvió a una pequeña muestra (representativa del tráfico pesado en la autovía) de 30 vehículos pesados, que previamente habían circulado sobre el sistema de pesaje dinámico a su velocidad normal de recorrido, hacia una báscula de pesaje estático perteneciente al Ministerio de Fomento, situada unos 500 m más allá, con objeto de contrastar los pesos suministrados por el sistema de pesaje dinámico con los dados por la báscula estática, que se pueden considerar como reales.

La calibración del sistema de pesaje dinámico se realizó simultáneamente a la toma de datos, pasando un cierto número de veces un camión de 2 ejes de peso conocido $(181,09 \mathrm{kN})$ sobre el sensor de peso del sistema de pesaje dinámico, de tipo capacitivo, a una velocidad próxima a la media de los vehículos pesados en la autopista (entre 80 y $90 \mathrm{~km} / \mathrm{h}$ ).

\subsection{Datos y cálculos previos realizados para la comprobación}

Respecto de los vehículos de la muestra que fueron desviados para su pesaje estático por la Guardia Civil, se obtuvieron los siguientes datos y cálculos inmediatos, que sirven de base para la comprobación:

1. Peso dinámico calibrado (Pdin) suministrado por el equipo de pesaje dinámico para cada uno de los vehículos.

2. Peso estático real (Pest) proporcionado por la báscula de pesaje estático para cada uno de los vehículos de la muestra.

3. Clasificación automática (en tipos) de los vehículos de la muestra a partir de los datos suministrados por el sistema de pesaje dinámico, mediante un programa desarrollado al efecto.

En la Tabla 1 se muestran todos estos datos y cálculos de cada vehículo de la muestra, incluyendo la hora de paso, el peso estático proporcionado por la báscula fija, el peso dinámico 
Tabla 1. Datos de los pesajes realizados en el ensayo: pesos estático, dinámico y error relativo en los pesos brutos de los vehículos de la muestra.

\begin{tabular}{|c|c|c|c|c|c|}
\hline Vehículo $\mathbf{n}^{\circ}$ & Hora de paso & Tipo de vehículo & $\begin{array}{l}\text { Peso estático } \\
(\mathrm{kN})\end{array}$ & $\begin{array}{c}\text { Peso dinámico } \\
(\mathrm{kN})\end{array}$ & Error relativo (\%) \\
\hline 1 & 10:06:18 & $\mathrm{T} 2 \mathrm{~S}_{3}$ & 378,37 & 362,97 & $-4,07$ \\
\hline 2 & 10:24:11 & $\mathrm{T} 2 \mathrm{~S}_{3}$ & 415,01 & 411,04 & $-0,96$ \\
\hline 3 & $10: 25: 30$ & $\mathrm{~T} 2 \mathrm{~S}_{3}$ & 281,55 & 301,17 & 6,97 \\
\hline 4 & 10:54:08 & $\mathrm{T} 2 \mathrm{~S}_{3}$ & 237,16 & 255,06 & 7,55 \\
\hline 5 & $10: 57: 32$ & $\mathrm{~T} 2 \mathrm{~S}_{3}$ & 341,83 & 337,46 & $-1,28$ \\
\hline 6 & $11: 25: 42$ & $\mathrm{~T} 2 \mathrm{~S}_{3}$ & 326,72 & 337,46 & 3,29 \\
\hline 7 & 11:35:16 & $\mathrm{C} 2$ & 113,35 & 135,38 & 19,43 \\
\hline 8 & 11:53:12 & $\mathrm{C} 2$ & 48,46 & 51,99 & 7,29 \\
\hline 9 & 12:08:59 & $\mathrm{T} 2 \mathrm{~S}_{3}$ & 320,79 & 305,09 & $-4,89$ \\
\hline 10 & $12: 15: 55$ & $\mathrm{~T}_{2} \mathrm{~S}_{3}$ & 219,20 & 244,27 & 11,43 \\
\hline 11 & 12:26:01 & $\mathrm{C} 2$ & 110,56 & 129,49 & 17,13 \\
\hline 12 & 12:41:11 & $\mathrm{T}_{2} \mathrm{~S}_{3}$ & 192,82 & 230,54 & 19,56 \\
\hline 13 & 12:49:54 & $\mathrm{T}_{2} \mathrm{~S}_{3}$ & 394,56 & 412,02 & 4,43 \\
\hline 14 & $10: 24: 30$ & $\mathrm{~T} 2 \mathrm{~S}_{3}$ & 418,64 & 376,70 & $-10,02$ \\
\hline 15 & 10:38:00 & $\mathrm{C} 2 \mathrm{R} 2$ & 305,68 & 309,02 & 1,09 \\
\hline 16 & 10:51:19 & $\mathrm{T}_{2} \mathrm{~S}_{3}$ & 388,82 & 383,57 & $-1,35$ \\
\hline 17 & 10:54:49 & $\mathrm{T}_{2} \mathrm{~S}_{3}$ & 292,83 & 330,60 & 12,90 \\
\hline 18 & 11:00:21 & $\mathrm{T}_{2} \mathrm{~S}_{3}$ & 414,42 & 393,38 & $-5,08$ \\
\hline 19 & 11:13:59 & $\mathrm{T}_{2} \mathrm{~S}_{3}$ & 239,81 & 254,08 & 5,95 \\
\hline 20 & $11: 14: 58$ & C2 & 122,33 & 142,25 & 16,28 \\
\hline 21 & 11:24:05 & $\mathrm{C}_{3}$ & 256,29 & 266,83 & 4,11 \\
\hline 22 & 11:38:15 & $\mathrm{T}_{2} \mathrm{~S}_{3}$ & 314,07 & 318,83 & 1,51 \\
\hline 23 & $11: 43: 38$ & $\mathrm{~T}_{2} \mathrm{~S}_{3}$ & 307,84 & 317,84 & 3,25 \\
\hline 24 & 11:53:14 & $\mathrm{C} 2$ & 143,81 & 164,81 & 14,60 \\
\hline 25 & 12:03:36 & $\mathrm{T}_{2} \mathrm{~S}_{3}$ & 394,75 & 412,02 & 4,37 \\
\hline 26 & 12:19:03 & $\mathrm{T}_{2} \mathrm{~S}_{3}$ & 403,58 & 411,04 & 1,85 \\
\hline 27 & 12:35:12 & $\mathrm{T}_{2} \mathrm{~S}_{3}$ & 408,05 & 426,74 & 4,58 \\
\hline 28 & 12:38:01 & $\mathrm{T}_{2} \mathrm{~S}_{3}$ & 250,30 & 312,94 & 25,02 \\
\hline 29 & 13:01:53 & $\mathrm{T}_{2} \mathrm{~S}_{3}$ & 403,83 & 464,01 & 14,90 \\
\hline 30 & $13: 17: 58$ & $\mathrm{~T}_{2} \mathrm{~S}_{3}$ & 229,85 & 254,08 & 10,54 \\
\hline
\end{tabular}

\begin{tabular}{|l|l|}
\hline Leyenda & \\
\hline $\mathrm{C} 2$ & Camión rígido de 2 ejes \\
\hline $\mathrm{C}_{3}$ & Camión rígido de 3 ejes \\
\hline $\mathrm{T} 2 \mathrm{~S} 2$ & Tractor de 2 ejes + semirremolque de 2 ejes \\
\hline $\mathrm{T} 2 \mathrm{~S} 3$ & Tractor de 2 ejes + semirremolque de 3 ejes \\
\hline $\mathrm{C} 2 \mathrm{R} 2$ & Camión rígido de 2 ejes + remolque de 2 ejes \\
\hline
\end{tabular}

\begin{tabular}{|c|}
\hline Error relativo medio (\%) \\
\hline 6,35 \\
\hline
\end{tabular}

\begin{tabular}{|c|}
\hline Desviación típica (\%) \\
\hline 8,41 \\
\hline
\end{tabular}

(ya corregido con el factor de calibración) suministrado por el equipo de pesaje dinámico y el error relativo obtenido en la medición del peso de cada uno de los vehículos.

\subsection{Cálculo de la exactitud y precisión del sistema de pesaje en movimiento}

Una vez obtenidos o determinados los anteriores datos o parámetros de los vehículos pesados, se realizaron los siguientes cálculos para determinar el sesgo y la dispersión de las medidas de los pesos proporcionadas por el sistema WIM:

1. Cálculo del error relativo para cada vehículo en la determinación de su peso bruto total, a través de realizar la siguiente operación:

$$
\text { Error relativo }(\%)=[(\text { Pdin }- \text { Pest }) / \text { Pest }] \cdot 100
$$

2. Determinación del valor medio de todos los errores relativos obtenidos para cada vehículo individual, obtenién- dose el llamado error relativo medio del pesaje dinámico para el conjunto de vehículos de la muestra, que representa el sesgo medio (\%) del sistema de pesaje dinámico.

El valor promedio de todos los errores relativos en el ensayo ha resultado ser del $6,35 \%$, valor que puede ser considerado como bastante mediocre, lo que puede deberse a que la calibración se ha realizado utilizando un solo vehículo (un camión de 2 ejes), como suele ser normal en los equipos portátiles de pesaje, utilizados para tomas de datos de corta duración.

3. Determinación de la desviación típica de los errores relativos obtenidos para cada vehículo, que representa la precisión del sistema de pesaje instalado en el firme de este emplazamiento.

El valor obtenido para la desviación típica ha sido del $8,41 \%$, que puede considerarse bastante bueno, pues es algo inferior al obtenido en el ensayo de referencia de sistemas portátiles de pesaje dinámico realizado en Trappes (Francia) en 1996 (6), lo que puede ser debido a que las 
condiciones del pavimento en el emplazamiento del ensayo eran muy buenas.

\section{5•3. Cálculo del factor de equivalencia}

Se han realizado los cálculos del factor de equivalencia, tanto en ejes de $128 \mathrm{kN}$ (antiguo límite legal de peso para los ejes simples en España) como en ejes de $113 \mathrm{kN}$ (actual límite legal en España), de cada vehículo de la muestra utilizando la fórmula del ensayo AASHO, tanto para un exponente 4 (firmes flexibles) como para un exponente 8 (firmes semirrígidos) y se ha hallado el factor de equivalencia medio por vehículo pesado en ambos casos, utilizando las dos series de datos siguientes:

- los pesos por eje de cada vehículo y las distancias entre los distintos ejes individuales (con objeto de determinar si forman un eje tándem o trídem) proporcionados por el sistema de pesaje dinámico (Tabla 3);

- los pesos obtenidos mediante pesaje estático (Tabla 2), que sirven de referencia.

Para el cálculo se ha utilizado la equivalencia de que cada eje tándem equivale a 1,4 ejes simples de peso $\mathrm{P} / 2$ y cada eje trídem equivale a 2,325 ejes simples de peso $\mathrm{P} / 3$. En las Tablas 2 y 3 se muestran los resultados de los cálculos del factor de equivalencia obtenidos con ambas series de datos (pesos estáticos y dinámicos).

Introduciendo estos valores en las fórmulas exponenciales de los deterioros, resultan los siguientes valores para las constantes $K_{T}$ y $K_{T r}$ de la expresión [3]:

Ejes tándem:

Para exponente $\alpha=4 \rightarrow K_{T}=0,0875$ para exponente $\alpha=8 \rightarrow K_{T}=0,0055$
Ejes trídem:

Para exponente $\alpha=4 \rightarrow K_{T r}=0,0287$
para exponente $\alpha=8 \rightarrow K_{T r}=0,00035$

\subsection{Discusión de resultados}

A la vista de los resultados puede comprobarse que el factor de equivalencia por vehículo pesado para un valor del exponente $\alpha=4$, calculado utilizando los pesos obtenidos mediante pesaje dinámico, ha sido de o,66 ejes equivalentes de $128 \mathrm{kN}$ por vehículo pesado, que ha resultado ser casi un $18 \%$ mayor que el que se ha obtenido utilizando los pesos medidos mediante básculas estáticas (0,56 ejes equivalentes de $128 \mathrm{kN}$ ). Para un valor del exponente $\alpha=8$, el factor de equivalencia medio obtenido mediante los pesajes en movimiento ha sido igualmente un $18 \%$ mayor que el que se ha obtenido con los pesajes estáticos (o,39 ejes equivalentes frente 0,33 mediante pesajes estáticos).

Por tanto, el error en el que se ha incurrido al determinar los pesos de los vehículos mediante pesaje dinámico, que como se ha visto ha tenido un sesgo de un $6,35 \%$ por exceso y una dispersión del $8,41 \%$, se ha magnificado al utilizar la expresión potencial obtenida en el ensayo AASHO, resultando un error del $18 \%$ por exceso en la determinación del coeficiente de equivalencia medio de los vehículos y, por tanto, del número total de aplicaciones de la carga tipo de $128 \mathrm{kN}$, lo que viene a significar que con ello se estaría infraestimando la vida útil del firme en ese porcentaje del $18 \%$, dado que ésta se estima en función del número de aplicaciones de la carga tipo que puede resistir el firme.

Sin embargo, el error total obtenido en la determinación de la vida de servicio es apreciablemente menor que el que podría estimarse por las consideraciones estadísticas dadas en (14), relativas al sesgo adicional introducido por el error relativo medio y la dispersión de las medidas del sistema de pesaje di-

Tabla 2. Factores de equivalencia calculados en el ensayo con los datos de los pesajes estáticos.

\begin{tabular}{|c|c|c|c|c|c|c|c|c|c|c|c|c|}
\hline \multicolumn{13}{|c|}{ Estación: Trijueque (Guadalajara) A-2 p.k. 82 Fecha: Junio 2015 Muestra de vehículos tomados por la Guardia Civil en báscula fija } \\
\hline \multicolumn{4}{|c|}{ Factor de equivalencia de vehículo pesado con ejes completos } & \multicolumn{2}{|c|}{ Eje tipo $=128 \mathrm{kN}$} & \multicolumn{2}{|c|}{ Eje tipo=113 kN } & \multirow[t]{2}{*}{$\begin{array}{c}\text { Silueta } \\
\text { calculada }\end{array}$} & \multicolumn{2}{|c|}{$\begin{array}{c}\Sigma \text { ejes } \\
\text { equivalentes } \\
(128 \mathrm{kN})\end{array}$} & \multicolumn{2}{|c|}{$\begin{array}{c}\Sigma \text { ejes } \\
\text { equivalentes } \\
(113 \mathrm{kN})\end{array}$} \\
\hline \multicolumn{2}{|c|}{ Tipo } & Silueta obtenida por elaboración Excel & Descripción & $a=4$ & $a=8$ & $a=4$ & $a=8$ & & $a=4$ & $a=8$ & $a=4$ & $a=8$ \\
\hline & 1 & & Ligeros & & & & & & & & & \\
\hline \multirow{8}{*}{ 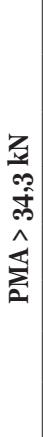 } & 2 & 吕 & Rígidos 2 ejes & 0,11 & 0,02 & 0,18 & 0,05 & 4 & 0,4 & 0,1 & 0,7 & 0,2 \\
\hline & 3 & 问 回 & Rígidos 3 ejes & 0,26 & 0,05 & 0,43 & 0,13 & 2 & 0,5 & 0,1 & 0,9 & 0,3 \\
\hline & 4 & 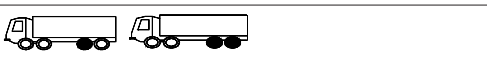 & Rígidos 4 ejes & & & & & & & & & \\
\hline & 5 & 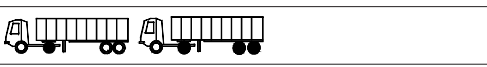 & Articulados 4 ejes & 0,72 & 0,31 & 1,18 & 0,84 & 1 & 0,7 & 0,3 & 1,2 & 0,8 \\
\hline & 6 & 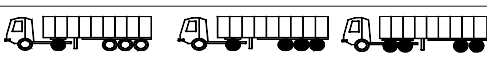 & Articulados 5 ejes & 0,66 & 0,41 & 1,08 & 1,10 & 23 & 15,2 & 9,5 & 24,8 & 25,2 \\
\hline & 7 & [9] & Articulados 6 ejes & & & & & & & & & \\
\hline & 8 & 可 $\longrightarrow$ & Trenes & & & & & & & & & \\
\hline & 9 & ○ & Autocares & & & & & & & & & \\
\hline \multicolumn{3}{|c|}{ Vehículos pesados en la población total } & & & & & & 30 & 16,9 & 10,0 & 27,5 & 26,5 \\
\hline \multicolumn{8}{|c|}{ Factor de equivalencia de vehículo pesado en el conjunto de la estación (cálculo con ejes completos) } & & & & & \\
\hline \multicolumn{6}{|c|}{\begin{tabular}{|l|l} 
Factor de equivalencia para $\alpha=4$ y eje tipo de $128 \mathrm{kN}$ \\
\end{tabular}} & \multicolumn{2}{|c|}{0,56} & & & & & \\
\hline \multicolumn{6}{|c|}{ Factor de equivalencia para $\alpha=8$ y eje tipo de $128 \mathrm{kN}$} & \multicolumn{2}{|c|}{0,33} & & & & & \\
\hline \multicolumn{6}{|c|}{ Factor de equivalencia para $\alpha=4$ y eje tipo de $113 \mathrm{kN}$} & \multicolumn{2}{|c|}{0,92} & & & & & \\
\hline \multicolumn{6}{|c|}{ Factor de equivalencia para $\alpha=8$ y eje tipo de $113 \mathrm{kN}$} & \multicolumn{2}{|c|}{0,88} & & & & & \\
\hline
\end{tabular}


Tabla 3. Factores de equivalencia calculados en el ensayo con los datos de los pesajes dinámicos.

\begin{tabular}{|c|c|c|c|c|c|c|c|c|c|c|c|c|}
\hline \multicolumn{13}{|c|}{ Estación: Trijueque (Guadalajara) A-2 p.k. 82 Fecha: Junio 2015 Muestra de vehículos tomados por la Guardia Civil en BDP } \\
\hline \multicolumn{4}{|c|}{ Factor de equivalencia de vehículo pesado con ejes completos } & \multicolumn{2}{|c|}{ Eje tipo $=128 \mathrm{kN}$} & \multicolumn{2}{|c|}{ Eje tipo=113 kN } & \multirow[t]{2}{*}{$\begin{array}{c}\text { Silueta } \\
\text { calculada }\end{array}$} & \multicolumn{2}{|c|}{$\begin{array}{c}\Sigma \text { ejes } \\
\text { equivalentes } \\
(128 \mathrm{kN})\end{array}$} & \multicolumn{2}{|c|}{$\begin{array}{c}\Sigma \text { ejes } \\
\text { equivalentes } \\
(113 \mathrm{kN})\end{array}$} \\
\hline \multicolumn{2}{|c|}{ Tipo } & Silueta obtenida por elaboración Excel & Descripción & $a=4$ & $a=8$ & $a=4$ & $a=8$ & & $a=4$ & $a=8$ & $a=4$ & $a=8$ \\
\hline & 1 & & Ligeros & & & & & & & & & \\
\hline \multirow{8}{*}{ 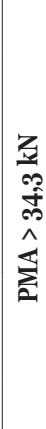 } & 2 & $\square$ & Rígidos 2 ejes & 0,20 & 0,05 & 0,33 & 0,13 & 5 & 1,0 & 0,2 & 1,7 & 0,7 \\
\hline & 3 & {$\left[\begin{array}{ll}\square & \square\end{array}\right.$} & Rígidos 3 ejes & 0,57 & 0,16 & 0,93 & 0,43 & 1 & 0,6 & 0,2 & 0,9 & 0,4 \\
\hline & 4 & 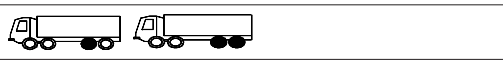 & Rígidos 4 ejes & & & & & & & & & \\
\hline & 5 & 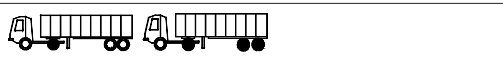 & Articulados 4 ejes & 0,64 & 0,21 & 1,04 & 0,57 & 1 & 0,6 & 0,2 & 1,0 & 0,6 \\
\hline & 6 & 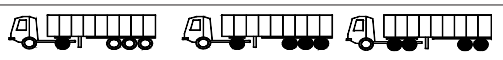 & Articulados 5 ejes & 0,76 & 0,49 & 1,24 & 1,30 & 23 & 17,5 & 11,2 & 28,6 & 29,8 \\
\hline & 7 & 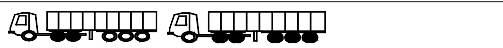 & Articulados 6 ejes & & & & & & & & & \\
\hline & 8 & प1 $\square \square$ & Trenes & & & & & & & & & \\
\hline & 9 & مण & Autocares & & & & & & & & & \\
\hline \multicolumn{3}{|c|}{ Vehículos pesados en la población total } & & & & & & 30 & 19,7 & 11,8 & 32,2 & 31,5 \\
\hline \multicolumn{8}{|c|}{ Factor de equivalencia de vehículo pesado en el conjunto de la estación (cálculo con ejes completos) } & & & & & \\
\hline \multicolumn{6}{|c|}{ Factor de equivalencia para $\alpha=4$ y eje tipo de $128 \mathrm{kN}$} & \multicolumn{2}{|c|}{0,66} & & & & & \\
\hline \multicolumn{6}{|c|}{ Factor de equivalencia para $\alpha=8$ y eje tipo de $128 \mathrm{kN}$} & \multicolumn{2}{|c|}{0,39} & & & & & \\
\hline \multicolumn{6}{|c|}{ Factor de equivalencia para $\alpha=4$ y eje tipo de $113 \mathrm{kN}$} & \multicolumn{2}{|c|}{1,07} & & & & & \\
\hline \multicolumn{6}{|c|}{ Factor de equivalencia para $\alpha=8$ y eje tipo de $113 \mathrm{kN}$} & \multicolumn{2}{|c|}{1,05} & & & & & \\
\hline
\end{tabular}

námico, debido a la incertidumbre de las medidas provocada por las oscilaciones inducidas en la suspensión de los vehículos por las irregularidades de los pavimentos, que vendrían dadas por la fórmula:

$$
\varepsilon=\left[(1+b)^{\alpha}-1\right]+\frac{\alpha(\alpha-1)}{2} \cdot(1+b)^{\alpha-2} \cdot \sigma^{2}
$$

Siendo:

$\varepsilon$ : error relativo medio en la estimación del deterioro total (o en la vida de servicio) del firme.

$b$ : error relativo medio de las mediciones del sistema de pesaje.

$\sigma:$ desviación típica de las medidas del sistema de pesaje.

$\alpha$ : exponente de la ley de fatiga ( $\alpha=4$ para firmes flexibles, $\alpha=8$ para firmes semirrígidos).

Así, si se entra en la fórmula anterior con los valores $b=$ 0,0635 y $\sigma=0,0841$, se obtendría que, por ejemplo, para firmes flexibles $(\alpha=4)$ el error en la vida de servicio del firme sería del 32,7\%, y la diferencia sería aún mayor para firmes semirrígidos.

Con objeto de ver la causa de esta diferencia, se ha realizado un breve análisis de sensibilidad del cálculo de los ejes equivalentes obtenidos en el ensayo, comprobándose que los valores resultantes son muy dependientes del error que se haya cometido al determinar los pesos dinámicos de los vehículos más cargados. Por ello, como los sistemas de pesaje dinámico normalmente determinan con mayor exactitud los pesos de los vehículos más cargados, el error realmente obtenido ha sido menor que el esperado.

\subsection{Representatividad estadística de los resultados}

Es siempre delicado tratar la representatividad estadística de los resultados obtenidos en muestras tan pequeñas, y más aún cuando los datos obtenidos en unas pocas horas de una misma semana se tratan de extrapolar al conjunto del tráfico pesado, que tiene unos ciclos de variación diario, semanal y estacional.

No obstante, se va a exponer, mediante la aplicación de las fórmulas de la inferencia estadística, la correspondencia entre las proporciones y características de los vehículos de la muestra con las de la población de vehículos en general, tanto de la misma carretera (la A-2, en Guadalajara) como del conjunto de la Red de Carreteras del Estado (RCE). Para ello se va a estimar un cierto intervalo de confianza para los parámetros de la población mediante una aplicación de las fórmulas de la inferencia estadística y se va a comparar con los resultados obtenidos en la última campaña de pesaje dinámico en la RCE, que tuvo lugar entre 2000 y 2003 y fue realizada por el CEDEX.

La comparación entre los datos obtenidos en la muestra del ensayo y la población se realizará con respecto a tres aspectos:

- Las proporciones de los distintos tipos de vehículos pesados.

- Los pesos medios de los vehículos pesados.

- Los factores de equivalencia medios por vehículo pesado.

En cuanto a las proporciones de los distintos tipos de vehículos, suele ser útil agrupar los distintos tipos de vehículos en las dos amplias clases que definen los dos tipos diferentes de transporte: por un lado, los vehículos rígidos (normalmente dedicados al reparto y al transporte de corto recorrido) y, por otro, los vehículos articulados (empleados para el transporte de medio y largo recorrido). Procediendo de esta forma con los camiones de la muestra, se tiene que 6 vehículos (un $20 \%$ ) son rígidos y 24 (un $80 \%$ ) son articulados.

Aplicando la fórmula del intervalo de confianza para las proporciones con los anteriores datos $(p=0,8, q=0,2)$ se obtie- 
ne que el intervalo de confianza al 95\% para las proporciones medias poblacionales estarían entre un $66 \%$ y un $94 \%$ para los articulados y entre un $6 \%$ y un $34 \%$ para los vehículos rígidos.

En la campaña 2000-2003 se realizó un pesaje en una estación muy cercana, en la A-2 pk 65,4, obteniéndose un porcentaje del $17 \%$ de vehículos rígidos y un $83 \%$ de articulados, mientras que para el conjunto de las 70 estaciones de la campaña el porcentaje de vehículos rígidos fue del 29,4\% y el de articulados un 70,6\%, por lo que se puede decir que los resultados de ambas poblaciones caen dentro del intervalo de confianza al $95 \%$.

En lo relativo al peso medio de los 30 vehículos pesados de la muestra, éste es de $289,18 \mathrm{kN}$, que aplicando la conocida fórmula [5], del intervalo de confianza para las medias, se obtiene que el intervalo de confianza al 95\% para la media de la población estaría entre $250,35 \mathrm{kN}$ y $328,04 \mathrm{kN}$.

$$
\mu=\bar{x} \pm t_{\mathrm{o}, 975} \cdot \frac{S}{\sqrt{n-1}}
$$

Por su parte, los resultados obtenidos en la campaña 20002003 para el emplazamiento cercano de la A-2 fue de 266,83 $\mathrm{kN}$, mientras que para el conjunto de las estaciones de la RCE fue de $254,08 \mathrm{kN}$, lo que quiere decir que en ambos casos el peso medio de la población estaba dentro del intervalo de confianza obtenido, aunque en el caso del conjunto de la campaña muy cerca del límite inferior.

En cuanto a la agresividad de los vehículos pesados en la muestra, el factor de equivalencia (calculado con los pesos estáticos) fue de 0,56 ejes equivalentes de $128 \mathrm{kN}$ para un exponente $\alpha=4$. Calculando el intervalo de confianza al 95\%, para el factor de equivalencia para $\alpha=4$, se obtiene que la agresividad media del tráfico pesado en la población debería estar entre 0,74 y 0,38 ejes equivalentes de $128 \mathrm{kN}$.

Como comparación, en la campaña 2000-2003 se obtuvo una agresividad media por vehículo pesado, en la A-2, de 0,51 ejes equivalentes de $128 \mathrm{kN}$ para un exponente $\alpha=4, \mathrm{y}$ 0,594 ejes equivalentes, también para $\alpha=4$, en el conjunto de las 70 estaciones de la campaña. Por tanto, se comprueba que ambos valores de agresividad están no sólo dentro del intervalo del confianza al 95\%, sino muy próximos a los valores medios obtenidos en la muestra del ensayo realizado, con lo cual se puede considerar que la muestra es bastante representativa de la población de vehículos, tanto para el tráfico pesado de la A-2, en las cercanías del emplazamiento, como en el conjunto de la RCE.

\section{INFLUENCIA DE LA PRECISIÓN DEL PESAJE EN MOVIMIENTO EN EL DIMENSIONAMIENTO MECÁNICO-EMPÍRICO DE FIRMES}

En la MEPDG (15) de la AASTHO se sigue un planteamiento basado en describir el tráfico en términos del número de ejes por tipos y las distribuciones de frecuencias de los pesos de cada tipo de eje, calculándose el grado de deterioro específico ocasionado por los ejes de cada peso. Por ello, numerosos autores se han centrado en evaluar la influencia de los errores en la determinación del espectro completo de cargas mediante pesaje en movimiento sobre la estimación de la vida de servicio de los firmes utilizando los principios mecánicoempíricos.
A este respecto puede destacarse el trabajo de Abbas et al. (18), que evaluó la diferencia en la estimación de la vida de servicio de varios diseños básicos de pavimentos flexibles de la MEPDG según se utilizaban diferentes espectros de cargas: los específicos para cada lugar obtenidos mediante pesaje dinámico o algunos dados por defecto en la norma. Tras el análisis se observó que los espectros dados por defecto en la MEPDG infraestimaban claramente la vida de servicio del pavimento (del orden del $50 \%$ ) y, por tanto, si se utilizaban para el dimensionamiento resultaban unos espesores de capas muy conservadores. Aparte se comprobó que el mecanismo de fallo más limitante y que, por tanto, controlaba el diseño del pavimento era la formación de roderas y no la fisuración por fatiga.

En otros estudios, uno llevado a cabo por SW. Haider et al. (19) y otro por R. Tarefder et al. (20), se investigó el impacto de los errores de los pesajes dinámicos (y, por tanto, de los espectros de cargas por eje) sobre el comportamiento de pavimentos tanto flexibles como rígidos, mostrando en ambos estudios que la fisuración es el deterioro más afectado por las variaciones en los espectros de cargas, tanto en firmes flexibles como rígidos, mientras que también queda afectada de forma moderada la formación de roderas en pavimentos flexibles y no observándose ningún efecto apreciable sobre la regularidad superficial.

\section{CONCLUSIONES}

En este documento se han expuesto los principales factores de influencia en la precisión de la técnica del pesaje de vehículos en movimiento, y se ha tratado de estimar el efecto que puede tener el error con el que son obtenidos los pesos de ejes y vehículos en la predicción de la vida de servicio de los firmes.

El factor que tiene más influencia en la precisión de los sistemas de pesaje dinámico es que lo que miden realmente estos equipos son las fuerzas de impacto de las ruedas de los vehículos sobre el pavimento, y estas fuerzas tienen una componente dinámica, debida principalmente a la excitación de la suspensión de los vehículos por las irregularidades del pavimento, que puede sobrepasar el $\pm 20 \%$ de la carga estática.

Con objeto de evaluar la influencia de la precisión de los pesajes dinámicos en las estimaciones de vida útil de los firmes se ha realizado un ensayo consistente en pesar una muestra de camiones tanto dinámica (mediante un sistema de pesaje en movimiento instalado en la carretera) como estáticamente, a efectos de comparación, obteniéndose que para unas mediciones dinámicas de una precisión entre media y baja (sesgo, 6,35\%; desviación típica 8,4\%) se incrementa el efecto sobre la exactitud en la estimación de la vida de servicio de los firmes, aproximadamente en un $18 \%$, tanto para firmes flexibles como semirrígidos, debido a la aplicación de la ley de potencias del ensayo AASHO.

Como comparación, diversos estudios utilizando el método mecánico-empírico de la Mechanistic-Empirical Pavement Design Guide de la AASTHO han mostrado que el efecto de un sesgo de los pesajes en la estimación de la vida de servicio de los firmes puede ser también bastante considerable, especialmente sobre la predicción de ciertos mecanismos de fallo, como la fisuración, tanto en firmes flexibles como rígidos, mientras que es menos evidente en la predicción de la formación de roderas. 


\section{REFERENCIAS}

(1) COST 323 Weigh-in-Motion of Road Vehicles (1999). European Specification on Weigh-in-Motion of Road Vehicles. EUCOCOST/323/8/99, LCPC, París.

(2) American Society for Testing and Materials (ASTM) (2009). ASTM E 1318-o9 Standard Specification for Highway Weigh-In-Motion (WIM) Systems with User Requirements and Test Method. American Society for Testing and Materials.

(3) Stanczyk, D., Jacob, B. (1998, 14-16 de septiembre). Essai europèen de systèmes de pesage en marche. Essai sur autoroute continentale (CMT), sur l'A-31. En Actes finaux de la 2ème Conference Européenne sur le pesage en marche des véhicules routiers. Lisboa: ECSC-EEC-EAEC, EUR 18488.

(4) Leal, J. (2012, 4-7 de junio). Evaluation of several piezoelectric WIM Systems. En Proceedings of the International Conference on Weigh-In-Motion ICWIM6 (pp. 218-227). Dallas: ISTE and John Wiley.

(5) Vaziri, SH., et al. (2013). Investigation of the effects of air temperature and speed on performance of piezoelectric weighin-motion systems. Canadian Journal of Civil Engineering, 40(10): 935-944, doi: http://dx.doi.org/10.1139/cjce-20120227.

(6) COST 323 Weigh-in-Motion of Road Vehicles (1998, noviembre). Portable and Multiple-Sensor WIM systems trial (Trappes, June 1996), Final Report. P. 57. COST and ISTU (Institut für Starssenbau und Starssenerhaltung). EUCOCOST $/ 323 / 10 / 98$.

(7) Lavric, I., Znidaric, A., Kalin, J. (2008, 18-22 de mayo). Improving bridge-WIM results with better road eveness and advanced compensations. En Proceedings of the International Conference on Heavy Vehicles-Weigh-in-Motion (ICWIM 5) (pp. 283-296). París: ISTE and John Wiley.

(8) Schmidt, F., Jacob, B. (2012, 4-7 de junio). Experimentation of a bridge WIM system in France and applications to bridge monitoring and overload screening. En Proceedings of the International Conference on Weigh-In-Motion ICWIM6 (pp. 33-42). Dallas: ISTE and John Wiley.

(9) OECD (1992). Vehicle loads on pavements and bridges. En Dynamic loading of pavements (pp. 21-40). París: Organisation for Economic Co-operation and Development (OECD-OCDE).

(10) Chatti, K., Lee, D. (2002, June 16-20). A profile based truck dynamic load index (DLI). En Proceedings of the 7 th International Symposium on Heavy vehicle Weights \& Dimensions (pp. 23-34). Delft, The Netherlands.

(11) Glover, M., Newton, W. (1991). Evaluation of a Multiple-sensor Weigh-In-Motion System, TRL Research Report 307. Crowthorne (UK): Transport and Road Research Laboratory.

(12) Ma, S., Caprez, M. (1995, 8-10 de marzo). The pavement roughness requirement for weigh-in-motion. En First European Conference on Weigh-in-Motion of Road Vehicles. Zurich: COST-ETH (Swiss Federal Institute of Technology).

(13) Del Val, M. A. (1993). Factores de dimensionamiento de los firmes. En Firmes y pavimentos I, IX Curso Internacional de Carreteras, Madrid: Fundación Agustín de Bethencourt.

(14) Management Committee of the COST 323 Action (2002). Pavement Applications. En COST 323 Weigh-in-Motion of Road Vehicles, Final report (1993-1998) (pp. 29-52). París: Laboratoire Central des Ponts et Chaussées.

(15) AASTHO (2008). Mechanistic-Empirical Pavement Design Guide. Interim Edition: A Manual of Practice. Estados Unidos: American Association of State Highway and Transportation Officials (AASTHO).

(16) Davies, V., Hathaway, P. J. (1998, 14-16 de septiembre). Étude de données de pesage en marche en Angleterre. En Actes finaux de la zème Conference Européenne sur le pesage en marche des véhicules routiers. Lisboa: ECSC-EEC-EAEC, EUR 18488.

(17) NCHRP. Transportation Research Board (1999). Guide for Mechanistic-Empirical Design of New and Rehabilitated Pavement Structures. Appendix AA: Traffic Loadings. Final Document. http://onlinepubs.trb.org/onlinepubs/archive/ mepdg/2appendices_AA.pdf.

(18) Abbas, A., et al. (2014). Effect of Traffic Load Input Level on Mechanistic-Empirical Pavment Design. Transportation Research Record , 2(2443): 63-77, doi: http://dx.doi.org/10.3141/2443-08.

(19) Haider, S. W., et al. (2010). Effect of Axle Load Measurement Errors on Pavement Performance and Design Reliability. Transportation Research Record 2160: 107-117, doi: http://dx.doi.org/10.3141/2160-12.

(20) Tarefder, R., Rodríguez-Ruiz, J. I. (2013). WIM data quality and its influence on predicted pavement performance. Transportation Letters -The International Journal of Transportation Research, 5(3): 154-163, doi: http://dx.doi.org/1 0.1179/1942786713Z.00000000017. 